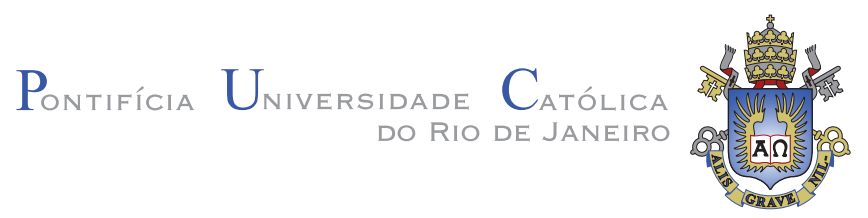

Ricardo Szczerbacki

\title{
Uso de Técnicas Baseadas em Pontos para Visualização de Horizontes Sísmicos
}

Dissertação de Mestrado

Dissertação apresentada ao Programa de Pós-graduação em Informática da PUC-Rio como requisito parcial para obtenção do título de Mestre em Informática.

Orientador: Prof. Marcelo Gattass 


\section{Pontifícia Universidade Católica $_{\text {a }}$

Ricardo Szczerbacki

\section{Uso de Técnicas Baseadas em Pontos para a Visualização de Horizontes \\ Sísmicos}

Dissertação apresentada como requisito parcial para a obtenção do grau de Mestre pelo Programa de Pósgraduação em Informática do Departamento de Informática do Centro Técnico e Científico da PUCRio. Aprovada pela Comissão Examinadora abaixo assinada.

\section{Prof. Marcelo Gattass}

Orientador

Departamento de Informática - PUC-Rio

Prof. Alberto Barbosa Raposo

Departamento de Informática - PUC-Rio

Prof. Anselmo Antunes Montenegro Universidade Federal Fluminense - UFF

Prof. José Eugenio Leal

Coordenador Setorial do Centro

Técnico Científico - PUC-Rio

Rio de janeiro, 10 de fevereiro de 2009 
Todos os direitos reservados. É proibida a reprodução total ou parcial do trabalho sem autorização da universidade, do autor e do orientador.

\section{Ricardo Szczerbacki}

Graduou-se em Informática na Universidade Federal do Rio de Janeiro (UFRJ). Hoje trabalha na Petrobras, onde atua em atividades relacionadas com a aplicação de computação científica à área de exploração e produção de petróleo.

Ficha Catalográfica

Szczerbacki, Ricardo

Uso de Técnicas Baseadas em Pontos para Visualização de Horizontes Sísmicos / Ricardo Szczerbacki; orientador: Marcelo Gattass. - Rio de Janeiro : PUC-Rio, Departamento de Informática, 2009.

v., 50 f: il. ; $29,7 \mathrm{~cm}$

1. Dissertação (mestrado) - Pontifícia Universidade Católica do Rio de Janeiro, Departamento de Informática.

Inclui referências bibliográficas.

1. Informática - Tese. 2. Visualização Científica. 3. Gráficos Baseados em Pontos. 4. Surface Splatting. 5. Horizontes Sísmicos.

I. Gattass, Marcelo. II. Pontifícia Universidade Católica do Rio de Janeiro. Departamento de Informática. III. Título. 


\section{Agradecimentos}

Ao Luiz Alberto, da Petrobras, por todo o incentivo e suporte.

Ao Fernando Rodrigues, da Petrobras, por sempre oferecer as oportunidades e condições para o meu desenvolvimento pessoal e profissional.

À Ivanise, da Petrobras, por todo o auxílio e atenção dispensados, nos últimos meses.

À Petrobras, por tornar possível esta realização.

Ao meu orientador, Professor Marcelo Gattass, pela paciência, pelo conhecimento compartilhado, pelo incentivo e apoio durante todo curso.

A todos os excepcionais professores da PUC-Rio, com quem tive o privilégio de aprender.

A todos os que tive a oportunidade de conhecer na Tecgraf, por todo o apoio.

Aos amigos e companheiros de mestrado e de Petrobras, Mario Pimenta e Righetto.

À minha família, por tudo. 


\title{
Resumo
}

\author{
Szczerbacki, Ricardo; Gattass, Marcelo. Uso de Técnicas Base- \\ adas em Pontos para Visualização de Horizontes Sísmicos. \\ Rio de Janeiro, 2009. 50p. Dissertação de Mestrado - Departamento \\ de Informática, Pontifícia Universidade Católica do Rio de Janeiro.
}

A visualização de horizontes sísmicos constitui uma importante área de conhecimento amplamente aplicada na prospecção de hidrocarbonetos pela indústria do petróleo. Diferentes técnicas são atualmente empregadas na apresentação destas superfícies, sendo usualmente utilizadas as soluções baseadas na geração de malhas poligonais, que se beneficiam da otimização das placas gráficas atuais no desenho de triângulos. Este trabalho faz uma avaliação do uso da renderização baseada em pontos, no lugar de polígonos, para a visualização de horizontes sísmicos. Para isso as técnicas de cada etapa do processo são avaliadas, levando-se em conta a natureza específica dos dados de interpretação de horizontes em volumes sísmicos e o resultado final esperado para a visualização deste tipo de dados. O algoritmo utilizado baseia-se no método conhecido como Surface Splatting para a renderização dos pontos originais, sendo estudados a estruturação apropriada para os dados a serem visualizados, a técnica para obtenção de normais, a abordagem adequada para o cálculo da iluminação e mecanismos adicionais necessários ao processo. Resultados da aplicação do método em dados reais são, ao final do trabalho, analisados e comparados à renderização tradicional para os horizontes avaliados.

\section{Palavras-chave}

Visualização Científica. Gráficos Baseados em Pontos. Surface Splatting. Horizontes Sísmicos. 


\section{Abstract}

Szczerbacki, Ricardo; Gattass, Marcelo (Advisor). Using Point Based Techniques for Seismic Horizons Visualization. Rio de Janeiro, 2009. 50p. MSc. Dissertation - Departamento de Informática, Pontifícia Universidade Católica do Rio de Janeiro.

Seismic horizon visualization stands as an important knowledge area used to support exploration on the oil industry. Different techniques currently employed to render this kind of surfaces are usually based on polygonal meshes generation, which benefits from graphics boards optimization on drawing triangles. This work is an evaluation of Point Based rendering techniques to replace polygonal approaches in seismic horizons visualization. To do so, this study revisits each stage of the seismic visualization process. The algorithm adopted here is based on the Surface Splatting with the EWA filter. This work also presents a study on normal evaluation and data structures to store points and normal. Special care is taken in shading techniques. The implementation yielded results that are used to support the evaluation of the Point Based Techniques on real 3D Seismic data. Traditional triangle based rendering is also presented to compare results.

\section{Keywords}

Scientific Visualization. Point Based Graphics. Surface Splatting. Seismic Horizons. 


\section{Sumário}

1 Introdução $\quad 12$

1.1 Sísmica de Reflexão 12

1.2 Horizontes Sísmicos $\quad 14$

1.3 Técnicas de Visualização $\quad 15$

1.4 Histórico da Visualização Baseada em Pontos 16

$\begin{array}{lll}1.5 & \text { Organização e Objetivos } & 18\end{array}$

2 Detalhamento do Processo Adotado $\quad 20$

2.1 Surface Splatting 20

2.2 Origem dos Dados 24

2.3 Organização de Dados 24

2.4 Frustum Culling 27

2.5 Back Face Splats 30

2.6 Cálculo de Normais 31

2.7 Estratégia de lluminação 34

2.8 Algoritmo Implementado 36

3 Resultados $\quad 40$

3.1 Distância versus Qualidade 41

3.2 Deferred Shading versus Shading por Splat 42

3.3 Pontos versus Poligonal 43

4 Conclusão $\quad 45$

4.1 Trabalhos Futuros $\quad 46$

$\begin{array}{ll}\text { Referências Bibliográficas } & 47\end{array}$ 


\section{Lista de figuras}

1.1 Esquema de levantamento sísmico marítimo 12

1.2 Cubo de dados sísmicos (Thomas04) 14

1.3 Horizontes em uma seção sísmica, adaptado de (Lyle02) 15

1.4 Horizonte sísmico 3D com seções sísmicas e poços de produção (Evans03)

1.5 Linha do tempo, com contribuições para a Visualização Baseada em Pontos, desde as primeiras abordagens.

2.1 Nuvem de pontos de um horizonte sísmico 3D interpretado (fundo do mar).

2.2 Parametrização 2D e área de influência de um conjunto de pontos, adaptado de (Zwicker01)

2.3 Aplicação de filtro de reconstrução

2.4 Exemplo de decomposição em blocos de uma octree e sua representação como árvore, onde as folhas brancas representam regiões sem pontos e as pretas regiões com pontos, adaptado de (Samet89) 25

2.5 Exemplo de uma kd-tree e sua representação como árvore, adaptado de (Samet89)

2.6 Exemplo de um frustum de visualização que contém apenas uma pequena parte de uma suprfície em sua região visível.

2.7 a) A bounding box está fora do view frustum; b) A bounding box está dentro do view frustum; c) A bounding box faz interseção com o view frustum.

2.8 a) Após a primeira iteração: são verificadas as bounding boxes dos 2 filhos do nó raiz; b) Após diversas iterações, onde as regiões em cinza são estão fora do frustum (serão descartadas), as regiões verdes estão dentro (serão processadas) e as regiões em azul têm interseção com o frustum (serão recursivamente iteradas).

2.9 a) centro do splat está fora do frustum, mas splat deveria ser desenhado; b) Após apliação do frustum, centro do splat passa a estar dentro do frustum.

2.10 Identificação da face do splat voltada para a câmera.

\section{5}

2.11 Plane fitting ponderado: busca de coeficientes do plano (A, B, C, D) que minimizam as distâncias dos pontos ao plano $\left(d_{1}, d_{2}, d_{3}\right.$ , ... ), através de otimização por mínimos quadrados, ponderados pela distância ao ponto central. Quanto maior a distância do ponto ao ponto central $\left(l_{12}, l_{13}, l_{14}, \ldots\right)$, menor o peso no cálculo. Coeficientes lineares do plano $(A, B, C)$ compõem a aproximação da normal no ponto central $d_{1}$.

2.12 Ponto central e sua vizinhança. Na figura da esquerda, os pontos estão distribuídos de forma regular. Na figura da direita a distribuição é irregular.

2.13 Normais calculadas para pontos de um horizonte sísmico interpretado 34

2.14 Para o splat central é encontrada a sua vizinhança, e então calculados o tamanho do splat e sua normal 
3.1 Visualização de $\mathrm{H1}$, de uma posição afastada 41

3.2 Visualização de $\mathrm{H} 1$, de uma posição próxima 41

3.3 Visualização de $\mathrm{H} 1$, de uma posição muito próxima 41

3.4 Visualização de $\mathrm{H} 2$, de uma posição afastada, utilizando deferred shading

3.5 Visualização de $\mathrm{H} 2$, de uma posição afastada, utilizando shading por splat

3.6 Visualização de $\mathrm{H} 2$, de uma posição próxima, utilizando deferred shading

3.7 Visualização de $\mathrm{H} 2$, de uma posição próxima, utilizando shading por splat

3.8 Visualização de H2, de uma posição afastada, utilizando Surface Splatting

3.9 Visualização de $\mathrm{H} 2$, de uma posição afastada, utilizando o método convencional

3.10 Visualização de H2, de uma posição próxima, com Surface Splatting 43

3.11 Visualização de $\mathrm{H} 2$, de uma posição próxima, com o método convencional 


\section{Lista de tabelas}

2.1 Estrutura do splat armazenado 24

2.2 Planos que delimitam o frustum 29

3.1 Arquivos XYZ utilizados 40

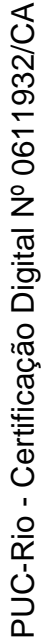

